\title{
The Optimizing Background Infusion Mode Decreases Intravenous Patient-Controlled Analgesic Volume and Opioid Consumption Compared to Fixed-Rate Background Infusion in Patients Undergoing Laparoscopic Cholecystectomy: A Prospective, Randomized, Controlled, Double-Blind Study
}

\author{
Ki Tae Jung ${ }^{1,2}$, Keum Young So ${ }^{1,2}$, Seung Un $\operatorname{Kim}^{2}$ and Sang Hun Kim ${ }^{1,2, *(D)}$ \\ 1 Department of Anesthesiology and Pain Medicine, School of Medicine, Chosun University, 309 Pilmun-daero, \\ Dong-gu, Gwangju 61452, Korea; mdmole@chosun.ac.kr (K.T.J.); kyso@chosun.ac.kr (K.Y.S.) \\ 2 Department of Anesthesiology and Pain Medicine, Chosun University Hospital, 365 Pilmun-daero, Dong-gu, \\ Gwangju 61453, Korea; sekizang@naver.com \\ * Correspondence: ksh3223@chosun.ac.kr; Tel.: +82-62-2203223
}

check for

updates

Citation: Jung, K.T.; So, K.Y.; Kim, S.U.; Kim, S.H. The Optimizing Background Infusion Mode Decreases Intravenous Patient-Controlled Analgesic Volume and Opioid Consumption Compared to Fixed-Rate Background Infusion in Patients Undergoing Laparoscopic Cholecystectomy: A Prospective, Randomized, Controlled, Double-Blind Study. Medicina 2021, 57, 42. https://doi.org/10.3390/ medicina57010042

Received: 11 December 2020 Accepted: 4 January 2021 Published: 6 January 2021

Publisher's Note: MDPI stays neutral with regard to jurisdictional clai$\mathrm{ms}$ in published maps and institutional affiliations.

Copyright: (C) 2021 by the authors. Licensee MDPI, Basel, Switzerland. This article is an open access article distributed under the terms and conditions of the Creative Commons Attribution (CC BY) license (https:// creativecommons.org/licenses/by/ $4.0 /)$.

\begin{abstract}
Background and objectives: The fixed-rate continuous background infusion mode with bolus dosing is a common modality for intravenous patient-controlled analgesia (PCA). However, some patients suffer from inadequate analgesia or opioid-related adverse effects due to the biphasic pattern of postoperative pain. Therefore, we investigated the postoperative analgesic efficacy of PCA using an optimizing background infusion mode (OBIM) where the background injection rate varies depending on the patient's bolus demand. Materials and Methods: We prospectively enrolled 204 patients who underwent laparoscopic cholecystectomy in a randomized, controlled, double-blind study. Patients were allocated to either the optimizing (group OBIM) or the traditional background infusion group (group TBIM). The numeric rating scale (NRS) score for pain was evaluated at admission to and discharge from the recovery room, as well as at the 6th, 24th, and 48th postoperative hours. Data on bolus demand count, total infused volume, and background infusion rate were downloaded from the PCA device at 30-min intervals until the 48th postoperative hour. Results: The NRS score was not significantly different between groups throughout the postoperative period $(p=0.621)$, decreasing with time in both groups $(p<0.001)$. The bolus demand count was not significantly different between groups throughout $(p=0.756)$. The mean total cumulative infused PCA volume was lower in group OBIM (84.0 (95\% confidence interval: $78.9-89.1) \mathrm{mL}$ ) than in group TBIM (102 (97.8-106.0) mL; $p<0.001)$. The total cumulative opioid dose in fentanyl equivalents, after converting sufentanil to fentanyl using an equipotential dose ratio, was lower in group OBIM (714.1 (647.4-780.9) $\mu \mathrm{g})$ than in group TBIM (963.7 (870.5-1056.9) $\mu \mathrm{g}) ; p<0.001)$. The background infusion rate was significantly different between groups throughout the study period $(p<0.001)$; it was higher in group OBIM than in group TBIM before the 12th postoperative hour and lower from the 18th to the 48th postoperative hour. Conclusions: The OBIM combined with bolus dosing reduces the cumulative PCA volume and opioid consumption compared to the TBIM combined with bolus dosing, while yielding comparable postoperative analgesia and bolus demand in patients undergoing laparoscopic cholecystectomy.
\end{abstract}

Keywords: background infusion; intravenous infusions; laparoscopic cholecystectomy; opioid analgesics; patient-controlled analgesia; postoperative pain

\section{Introduction}

Intravenous patient-controlled analgesia (PCA) is a common method of immediately delivering analgesics on an as-required basis to the patient via an infusion pump [1]. Its main benefit is the provision of appropriate analgesia according to patient demand, ultimately increasing patient satisfaction $[2,3]$. The most common PCA modes are intermittent, 
fixed demand dosing (self-administering) with or without continuous background infusion for postoperative analgesia $[4,5]$. Other variable parameters associated with PCA include the loading dose, bolus dose, lockout interval (time lag between bolus doses), and continuous background infusion rate [1].

However, despite using PCA devices, some patients experience inadequate analgesia due to the biphasic pattern of postoperative pain; it is more intense than anticipated immediately after surgery and less intense from the day after surgery [6,7]. Hence, patients may suffer from insufficient analgesia immediately after surgery, and may ultimately require frequent additional rescue analgesics because of the lockout interval and the fixed rate of a continuous background infusion [6,7]. They may also experience postoperative opioid-related adverse effects due to the combination of a self-administered bolus and fixed-rate continuous background infusion [8,9].

To address these shortcomings, the PAINSTOP medicine-injection pump (PS-1000, Unimedics Co., Ltd., Seoul, Korea) was introduced as a new PCA device providing an "optimizing background infusion mode" (OBIM) defined by the manufacturer [10]. The OBIM, also termed the "variable-rate feedback infusion mode" (VFIM), refers to the background injection rate that varies depending on bolus demand over a predefined time [1]. However, this mode is yet to be applied in clinical practice for postoperative pain control, and there is a lack of evidence of its utility $[1,10,11]$.

We hypothesized that the OBIM would provide better postoperative analgesia and a lower cumulative opioid consumption compared to the traditional (fixed-rate) background infusion mode (TBIM). To investigate this, we evaluated the efficacy of PCA in patients undergoing laparoscopic cholecystectomy using bolus dosing and either the OBIM or the TBIM for postoperative analgesia. The primary outcome of this study was achievement of a medium effect size (0.5) in the numeric rating scale (NRS) pain score sixhours postoperatively.

\section{Materials and Methods}

\subsection{Study Design and Ethical Statement}

This prospective, randomized, controlled, double-blind study was approved by the Institutional Review Board of Chosun University Hospital (Chosun 2018-02-011) on 6 March 2018, and was prospectively registered with the Clinical Research Information Service (CRIS: https: / / cris.nih.go.kr/, ref: KCT0002777) on 5 April 2018. It was conducted according to the Declaration of Helsinki of 1964 and all of its subsequent revisions.

\subsection{Selection of Study Population}

The subjects included patients aged 20 to 70 years with an American Society of Anesthesiologists (ASA) physical status of I-III who were scheduled to undergo elective laparoscopic cholecystectomy under general anesthesia between 3 September 2018 and 14 February 2020. Written informed consent was obtained from all participants after a thorough explanation of the purpose of the study. Participants were instructed to push the "demand" button of the PAINSTOP device whenever they experienced pain of $>4$ points on the numeric rating scale (NRS: $0=$ no pain, $10=$ worst pain imaginable). We excluded patients with severe cardiopulmonary disease, renal or hepatic functional abnormalities, neuromuscular disorders, or a history of opioid-related complications.

\subsection{Randomization and Masking}

Two hundred four patients were randomly assigned to two groups that used a PCA device applying either the fixed-rate background infusion mode (group TBIM, $n=102$ ) or the optimizing background infusion mode (group OBIM, $n=102$ ). In addition, the enrolled patients in each of these two groups were assigned randomly to one of two further groups according to whether they received fentanyl or sufentanil. Randomization was performed using a computer-generated table of random numbers via the permuted block method (a 
1:1 allocation ratio and a block size of 2). This randomization was performed using PASS 15 Power Analysis and Sample Size Software (2017) (NCSS, LLC., Kaysville, UT, USA).

The researcher who managed the anesthesia (RA) was responsible for obtaining informed consent from participants, as well as for gathering and recording data from the participants and the PCA devices. The researcher who managed the PCA (RP) was responsible for assigning the correct drugs to each PCA device according to the randomization scheme. For blinding, the RP recorded the drug assignment in the anesthesia charts after the anesthesia was finished, and the RA finally collated the data of patient medical records that were generated through the trial for at least $48 \mathrm{~h}$ postoperatively. The nurses in the recovery room (RR) or ward recorded postoperative pain and postoperative nausea and vomiting (PONV) using the NRS; these nurses were not part of the investigating team and were trained in the hospital to assess pain intensity and PONV with the NRS. Neither RA nor RP participated in the statistical analysis.

\subsection{Interventions}

After premedication with intramuscular midazolam $(0.05 \mathrm{mg} / \mathrm{kg})$, the patients were transported to an operating room. AA anesthetized the patients using total intravenous anesthesia with propofol and remifentanil and maintained the optimal neuromuscular paralysis with rocuronium. Ten minutes before the end of surgery, RP commenced the PCA device according to the group allocation, after administration of an initial bolus dose ( $2 \mathrm{~mL}$; fentanyl: $0.29 \mu \mathrm{g} / \mathrm{kg}$ or sufentanil $0.04 \mu \mathrm{g} / \mathrm{kg}$ ) from the PCA device and ramosetron $(0.3 \mathrm{mg})$. We used an equianalgesic dose of sufentanil and fentanyl (fentanyl:sufentanil = 6:1) [12].

The total PCA volume was $140 \mathrm{~mL}$, comprised of normal saline, fentanyl $(20 \mu \mathrm{g} / \mathrm{kg})$ or sufentanil $(3 \mu \mathrm{g} / \mathrm{kg})$, nefopam $(160 \mathrm{mg})$, and ramosetron $(1.2 \mathrm{mg})$. All PCA devices were initially set to administer a bolus of $2 \mathrm{~mL}$ (fentanyl: $0.29 \mu \mathrm{g} / \mathrm{kg}$ or sufentanil: $0.04 \mu \mathrm{g} / \mathrm{kg}$ ) with a lockout interval of $10 \mathrm{~min}$ and a background infusion rate of $2 \mathrm{~mL} / \mathrm{h}$. The background infusion rate of group OBIM was set to increase automatically by $0.4 \mathrm{~mL} / \mathrm{h}$ (fentanyl: $0.06 \mu \mathrm{g} / \mathrm{kg} / \mathrm{h}$ or sufentanil: $0.01 \mu \mathrm{g} / \mathrm{kg} / \mathrm{h}$ ) each time a bolus dose was required, and decrease by $0.2 \mathrm{~mL} / \mathrm{h}$ (fentanyl: $0.029 \mu \mathrm{g} / \mathrm{kg} / \mathrm{h}$ or sufentanil: $0.004 \mu \mathrm{g} / \mathrm{kg} / \mathrm{h}$ ) when a bolus dose was not required for $1.5 \mathrm{~h}$. The background infusion rate was limited to a maximum of $4.0 \mathrm{~mL} / \mathrm{h}$ (fentanyl: $0.57 \mu \mathrm{g} / \mathrm{kg} / \mathrm{h}$ or sufentanil: $0.09 \mu \mathrm{g} / \mathrm{kg} / \mathrm{h}$ ) and a minimum of $1 \mathrm{~mL} / \mathrm{h}$ (fentanyl: $0.14 \mu \mathrm{g} / \mathrm{kg} / \mathrm{h}$ or sufentanil: $0.02 \mu \mathrm{g} / \mathrm{kg} / \mathrm{h}$ ). All drug doses were based on the ideal body weight of patients. By comprehensively incorporating the opinions of manufacturers and researchers, due to lack of evidence for specific OBIM settings, we created final settings to ensure patient safety and to provide effective analgesia.

At the end of surgery, the patients did not receive any wound anesthetic infiltration with local anesthetics or any regional analgesia. The patients were transferred to the RR after the complete reversal of rocuronium-induced neuromuscular paralysis and when they were fully awake. When patients experienced pain in the RR of $>4$ points on the NRS, the RR nurse or the patient pushed the PCA button for administration of a bolus dose. When patients required additional rescue analgesics within the lockout interval, the RR nurse intravenously administered either ketorolac $(30 \mathrm{mg})$ or nefopam $(20 \mathrm{mg})$. We also allowed the intravenous injection of opioids, nonsteroidal anti-inflammatory drugs, or tramadol as rescue analgesics in the ward to treat pain of $>4$ points on the NRS. We treated PONV of $>4$ points on the NRS with the intravenous injection of metoclopramide (10 mg). Our research staff decided whether to stop the PCA device or change its settings based on the severity of signs and symptoms, and we excluded cases where this occurred from the final statistical analysis.

\subsection{Outcomes}

We recorded an NRS score for pain; PONV; and the need for additional rescue analgesics and antiemetics on admission to (RR1) and discharge from (RR2) the RR, as well as at the 6th, 24th, and 48th postoperative hours. We downloaded the data from the PCA device 
(bolus demand count, total infused volume, background infusion rate), using its built-in Wi-Fi system, in 30-min intervals until the 48th postoperative hour. We recorded data on demographics (age, sex, height, weight, ASA physical status, intraoperative remifentanil dose, operating time, anesthesia time, PCA composition) and perioperative complications, as well as the incidence of and causes for early termination of the PCA device.

\subsection{Sample Size}

To estimate the sample size for the primary outcome, we used G*Power software (ver. 3.1.9.1, Heinrich-Heine-Universität, Düsseldorf, Germany). We set the two-tailed level of statistical significance as $\alpha=0.05$, the power as $90 \%$, and the medium effect size as 0.5 (defined by Cohen for analyses using the Student $t$-test); the latter was an assumption, as there were no previous data from which to calculate the effect size [13].

The study required 172 patients in total; thus, we enrolled 204 patients, allowing for a dropout rate of approximately $15 \%$.

\subsection{Analysis}

IBM SPSS Statistics for Windows, ver. 26.0 (IBM Corp., Armonk, NY, USA) was used for all statistical analyses. All data were analyzed as if their probability distributions were normal based on the central limit theorem and are presented as means (95\% confidence intervals (CI)), means \pm standard deviation (SD), numbers (of patients $(n)$, or numbers (percentage) of patients $(n[\%]))$. We analyzed continuous variables using the Student $t$-test and nominal variables with the $\chi^{2}$ or Fisher's exact test. For analysis of time-interval data that passed Mauchly's sphericity test, we used repeated measures analysis of variance; for data that did not pass Mauchly's sphericity test, we used Wilk's lambda multivariate analysis of variance. To compare two groups in a given time interval, the Student $t$-test was used. $p$ values $<0.05$ were considered statistically significant.

\section{Results}

\subsection{Demographic Data}

There were no important harms or unintended effects in either group in this study. We enrolled 204 patients finally; however, 71 patients were excluded from the final analysis, representing a $34.8 \%$ dropout rate (Table 1, Figure 1).

Table 1. Incidences and causes for exclusion and early termination of PCA.

\begin{tabular}{cccc}
\hline Variables & Group TBIM $(\boldsymbol{n = 1 0 2 )}$ & Group OBIM $(\boldsymbol{n = 1 0 2 )}$ & $p$ Value \\
\hline Exclusion (No/Yes) & $79(77.5) / 23(22.5)$ & $54(52.9) / 48(47.1)$ & $<0.001$ \\
Causes for Exclusion & $13(12.7)$ & $25(24.5)$ & \\
Data loss & $7(6.9)$ & $13(12.7)$ & \\
Early PCA & $3(2.9)$ & $10(9.8)$ & \\
termination & $95(93.1) / 7(6.9)$ & $89(87.3) / 13(12.7)$ & \\
Setting error & & & \\
\hline Early PCA & & $2(2.0)$ & \\
termination (No/Yes) & $0(0.0)$ & $11(10.8)$ & \\
Causes for PCA & $7(6.9)$ & & \\
termination & Nausea & & \\
No pain & & & \\
\hline
\end{tabular}

Values are expressed as the number (percentage) of patients. PCA: patient-controlled analgesia, OBIM: optimizing background infusion mode, TBIM: traditional background infusion mode. 


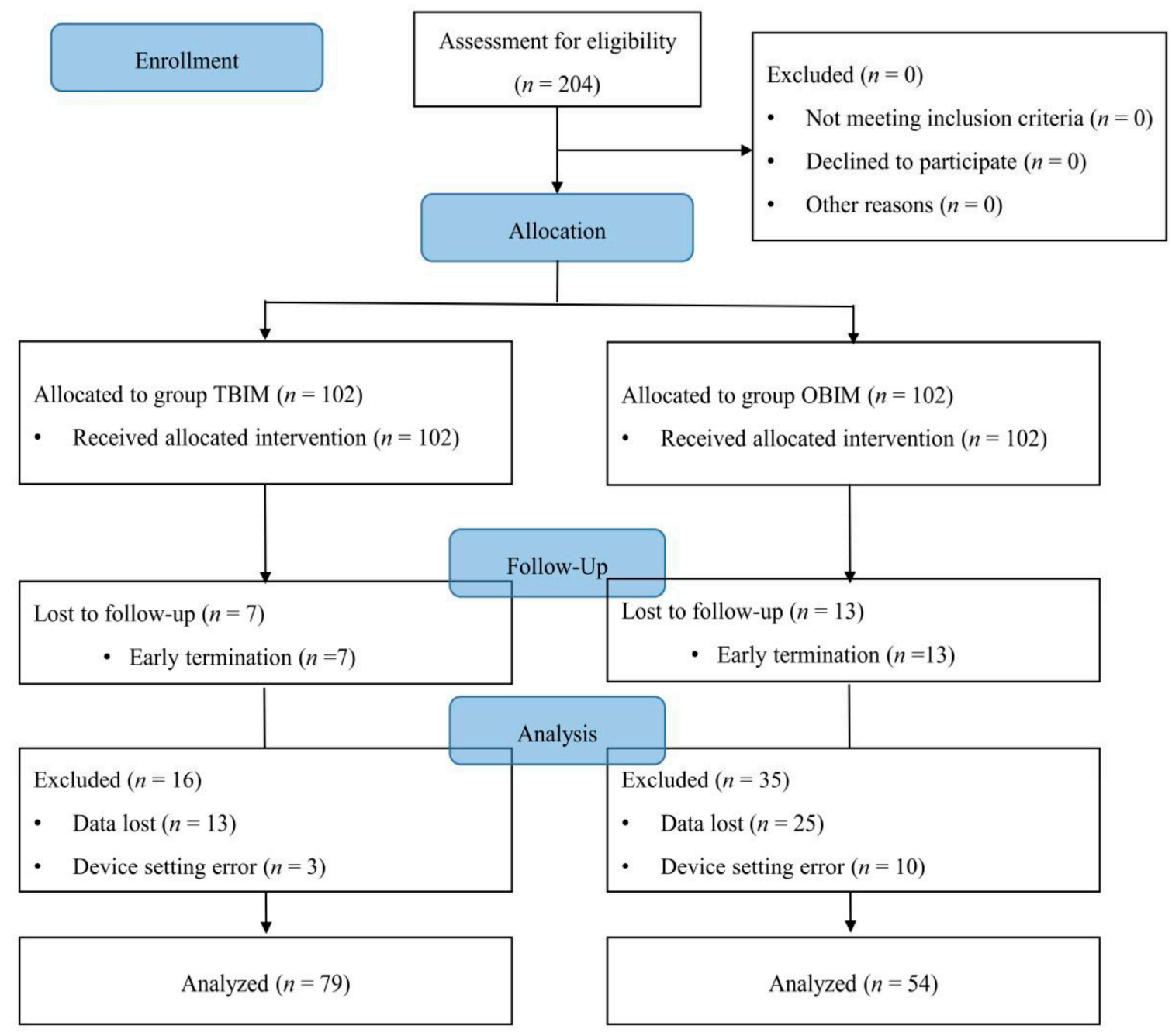

Figure 1. CONSORT diagram for patient recruitment. OBIM: optimizing background infusion mode, TBIM: traditional background infusion mode.

The number of excluded patients was significantly different between the groups $(p<0.001): 23(22.5 \%)$ in group TBIM and $48(47.1 \%)$ in group OBIM (Table 1$)$. The reasons for exclusion included data loss during collection in 38 patients $(18.6 \%)$, early PCA termination in 20 patients $(9.8 \%)$, and device setting errors in 13 patients (6.4\%; Table 1$)$.

The causes of early PCA termination were postoperative nausea (two patients in group OBIM) and patient request due to a lack of pain (seven in group TBIM, 11 in group OBIM). However, the number of early PCA terminations was not significantly different between the groups $(p=0.214$; Table 1$)$.

No statistically significant differences were observed in demographic data, intraoperative variables, or PCA regimens after exclusion of the above patients (Tables 2 and 3). The opioids (fentanyl and sufentanil) used in the PCA devices were not significantly different between the groups (fentanyl: 44 patients $(55.7 \%)$ and 23 patients $(42.6 \%)$ in groups TBIM and OBIM, respectively; $p=0.160$ ). 
Table 2. Demographic data and intraoperative variables.

\begin{tabular}{cccc}
\hline Variables & Group TBIM $(\boldsymbol{n}=\mathbf{7 9 )}$ & Group OBIM $(\boldsymbol{n}=\mathbf{5 4})$ & $\boldsymbol{p}$ Value \\
\hline Age $(\mathrm{y}$ & $49.7 \pm 12.3$ & $49.1 \pm 12.7$ & 0.795 \\
Sex (M/F) & $39 / 40$ & $34 / 20$ & 0.122 \\
Height (cm) & $165.5 \pm 8.4$ & $166.3 \pm 8.2$ & 0.589 \\
Weight (kg) & $68.7 \pm 13.6$ & $68.4 \pm 14.9$ & 0.888 \\
ASA-PS (I/II/III) & $39 / 36 / 4$ & $31 / 23 / 0$ & 0.203 \\
Cumulative remifentanil ( $\mu \mathrm{g})$ & $397.9 \pm 355.8$ & $369.9 \pm 303.9$ & 0.638 \\
Operation time (min) & $45.8 \pm 45.0$ & $43.9 \pm 33.5$ & 0.789 \\
Anesthesia time (min) & $59.1 \pm 46.5$ & $54.2 \pm 33.5$ & 0.503
\end{tabular}

Values are expressed as the means \pm standard deviation or number of patients. ASA-PS: American Society of Anesthesiologists physical status, OBIM: optimizing background infusion mode, TBIM: traditional background infusion mode.

Table 3. PCA regimens.

\begin{tabular}{cccc}
\hline Drugs & Group TBIM $(\boldsymbol{n}=\mathbf{7 9})$ & Group OBIM $(\boldsymbol{n}=\mathbf{5 4})$ & $\boldsymbol{p}$ Value \\
\hline Fentanyl $(\mu \mathrm{g})$ & $1195.5 \pm 180.7(n=44)$ & $1276.5 \pm 172.4(n=23))$ & 0.081 \\
Sufentanil $(\mu \mathrm{g})$ & $181.8 \pm 34.8(n=35)$ & $169.1 \pm 35.9(n=31)$ & 0.149 \\
Nefopam $(\mathrm{mg})$ & $160.0 \pm 0.0(160.0-160.0)$ & $160.0 \pm 0.0$ & 1.000 \\
Ramosetron $(\mathrm{mg})$ & $1.2 \pm 0.0$ & $1.2 \pm 0.0$ & 1.000 \\
\hline
\end{tabular}

Values are expressed as means \pm standard deviation. PCA: patient-controlled analgesia, OBIM: optimizing background infusion mode, TBIM: traditional background infusion mode.

\subsection{NRS Scores}

The NRS score was not significantly different between the groups throughout the postoperative period $(p=0.621)$, and it decreased with time in both groups $(p<0.001$, Figure 2).

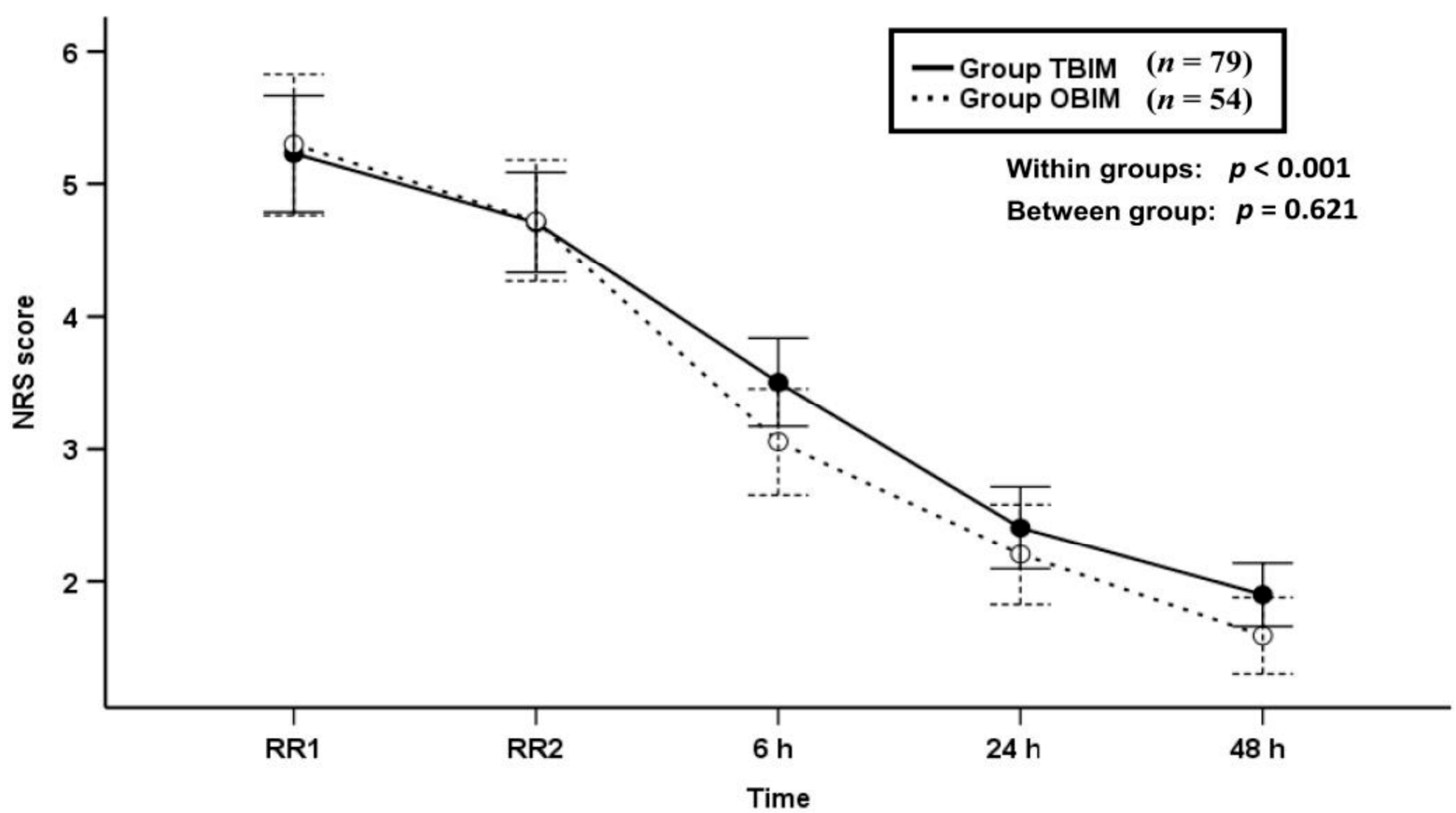

Figure 2. Time-sequential changes of numeric rating scale (NRS) scores. Data points and error bars represent means and $95 \%$ confidence intervals, respectively. OBIM: optimizing background infusion mode, TBIM: traditional background infusion mode, RR1: at admission from the recovery room, RR2: at discharge from the recovery room. 


\subsection{Bolus Demand Counts}

The bolus demand count was not significantly different between groups throughout the postoperative period ( $p=0.756$, Figure 3 ).

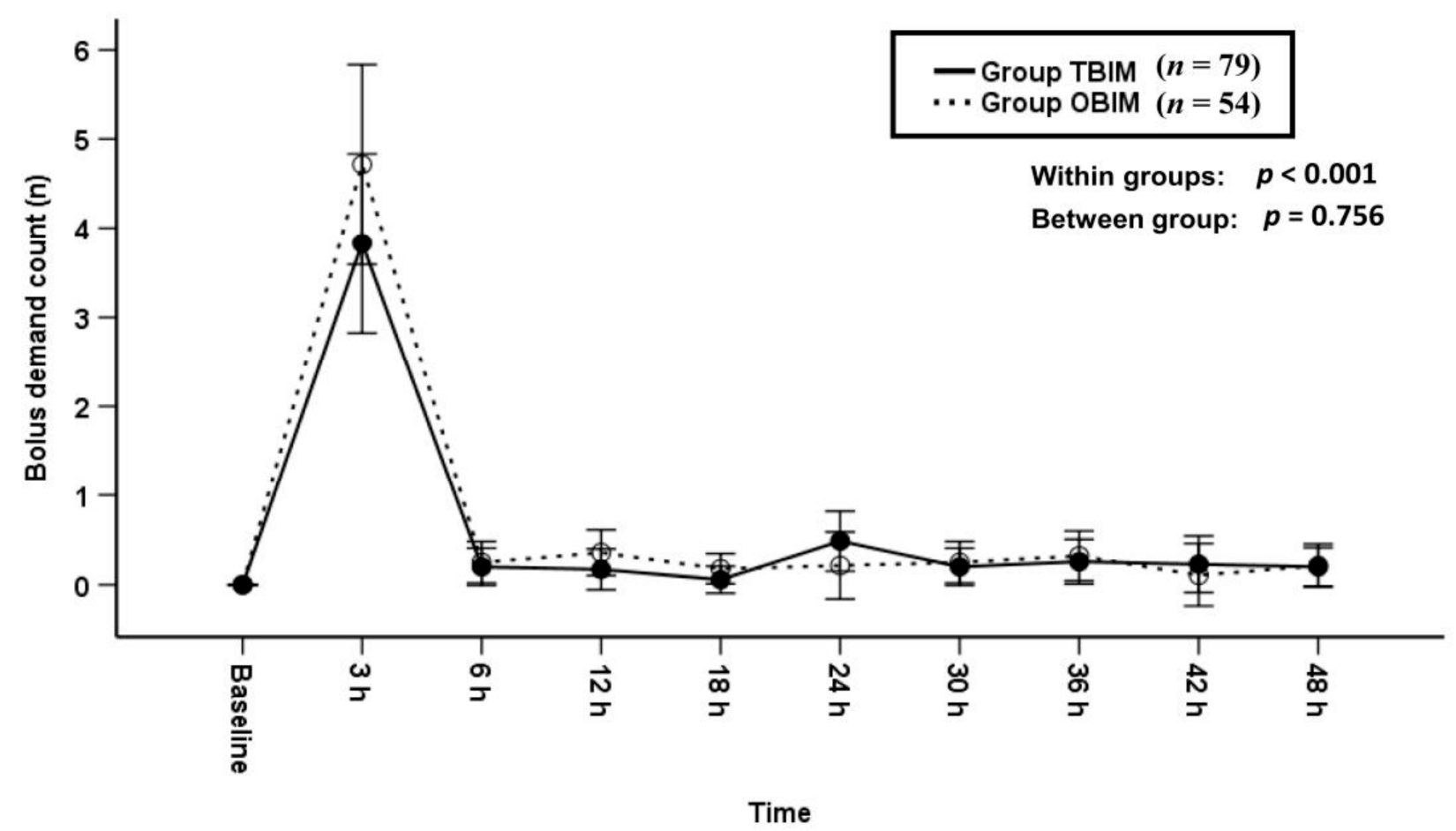

Figure 3. Time-sequential changes of bolus demand counts. Data points and error bars represent means and $95 \%$ confidence intervals, respectively. OBIM: optimizing background infusion mode, TBIM: traditional background infusion mode.

\subsection{Background Infusion Rate}

The background infusion rate was significantly different between groups throughout the postoperative period $(p<0.001$, Figure $4 a, b)$. The background infusion rate of group OBIM was significantly different from that of group TBIM for all time intervals except for the 12th postoperative hour ( $p<0.001$, Figure $4 \mathrm{~b}$ ). The background infusion rate was higher in group OBIM than in group TBIM before the 12th postoperative hour and lower from the 18th to the 48th hours (Figure $4 \mathrm{~b}$ ). The maximum and minimum background infusion rates were $3.3(3.2-3.5)$ and $1.1(1.0-1.2) \mathrm{mL} / \mathrm{h}$, respectively in group OBIM, while the background infusion rate in group TBIM was constant at $2.0 \mathrm{~mL} / \mathrm{h}$. 




(a)

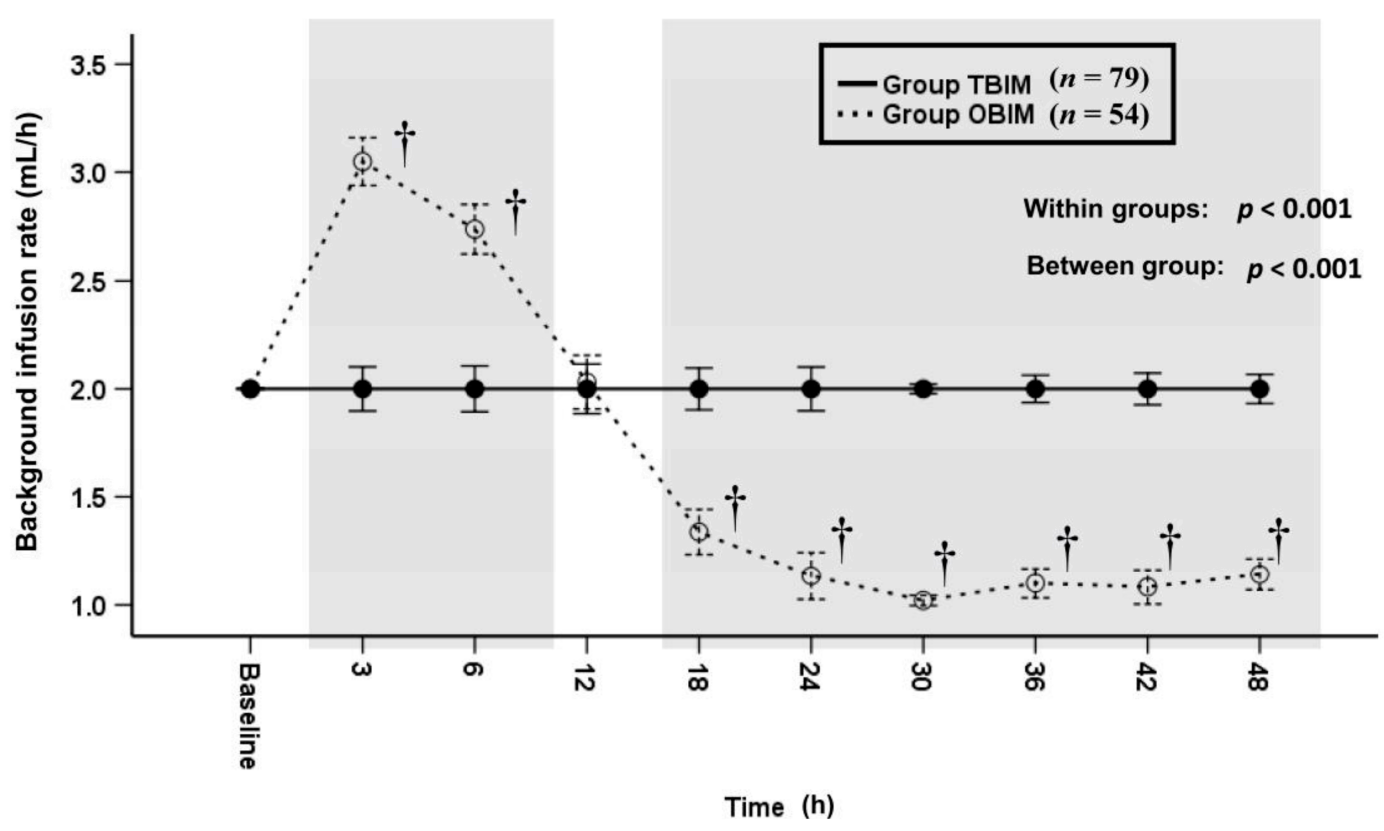

(b)

Figure 4. Time-sequential changes of background infusion rate at 30-min intervals (a) and at specific time points (b). Data points and error bars represent means and 95\% confidence intervals, respectively. OBIM: optimizing background infusion mode, TBIM: traditional background infusion mode. $+: p<0.001$ compared with group TBIM.

\subsection{Infused PCA Volumes and Infused Opioid Doses}

The cumulative infused PCA volume was significantly different throughout the postoperative period $(p<0.001)$ and at each measured interval $(p \leq 0.005)$ except at the 24th and 30th postoperative hours (Figure 5a). It was higher in group OBIM than in group TBIM until the 18th postoperative hour and lower from the 38th to the 48th postoperative 
hour (Figure 5a). The total cumulative infused volume was lower in group OBIM ( 84.0 $(78.9-89.1) \mathrm{mL})$ than in group TBIM $(102(97.8-106.0) \mathrm{mL}$; mean difference $(95 \% \mathrm{CI}): 17.9$ (11.6 to 24.2), $p<0.001$, Figure 5a). The per-interval infused PCA volume was significantly different between groups throughout the postoperative period $(p<0.001$, Figure $5 b)$; it was higher in group OBIM than in group TBIM until the 12th postoperative hour and lower from the 24th to the 48th hours ( $p \leq 0.004$, Figure $5 b$ ).

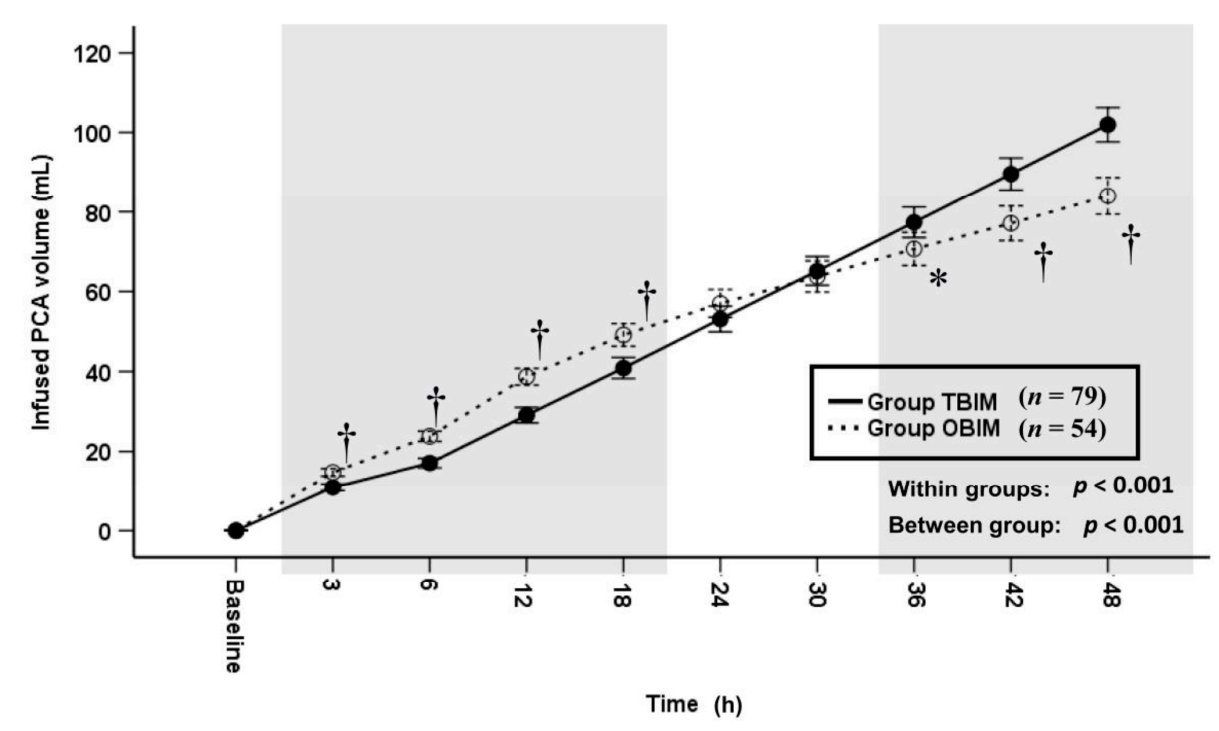

(a)

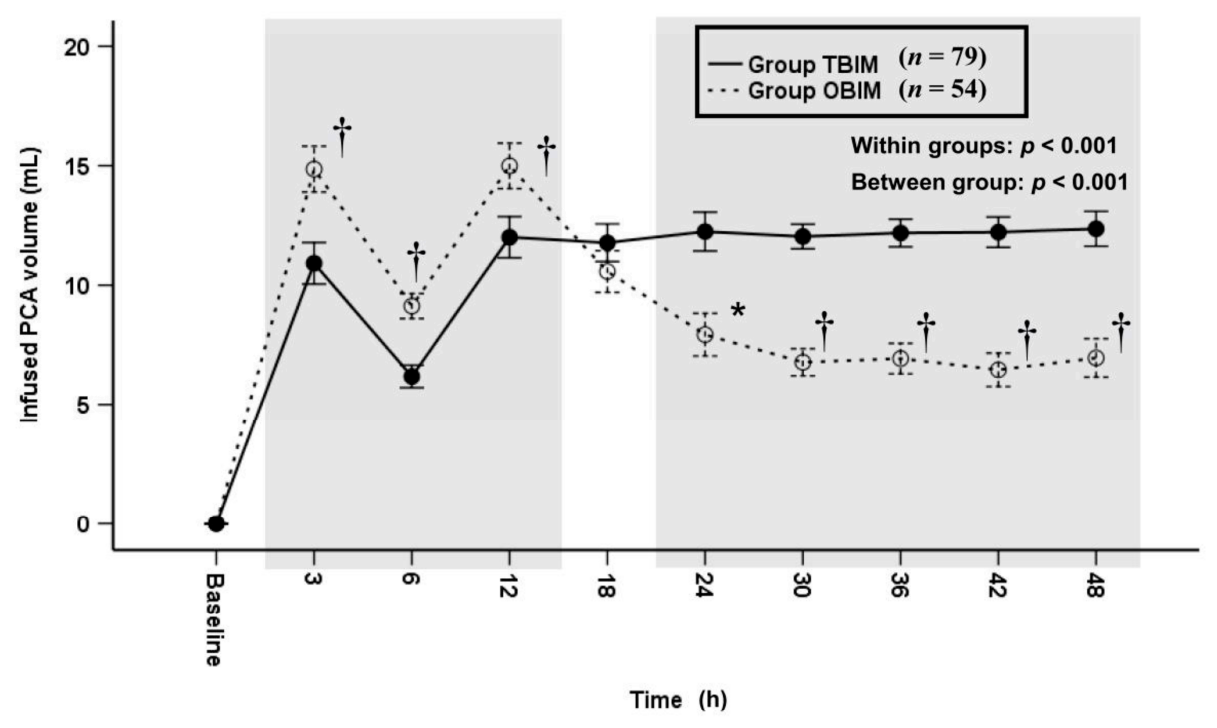

(b)

Figure 5. Time-sequential changes of cumulative (a) and per-interval (b) infused PCA volumes. The gray boxes represent the intervals in which there were statistically significant differences between the groups. Data points and error bars represent means and 95\% confidence intervals, respectively. OBIM: optimizing background infusion mode, PCA: patient-controlled analgesia, TBIM: traditional background infusion mode. * $p<0.05$ compared with group TBIM, $+: p<0.001$ compared with group TBIM.

In addition, we analyzed differences of the cumulative and per-interval infused opioid doses between the two groups after the sufentanil doses were converted to opioid doses in fentanyl equivalents. The cumulative infused opioid dose was significantly different 
throughout the postoperative period $(p<0.001)$ and at each measured interval $(p \leq 0.006)$ except at the 18th, 24th and 30th postoperative hours (Figure 6a). It was higher in group OBIM than in group TBIM until the 12th postoperative hour and lower from the 38th to the 48th postoperative hour (Figure 6a). The total cumulative opioid dose was lower in group OBIM (714.1 (647.4-780.9) $\mu \mathrm{g})$ than in group TBIM (963.7 (870.5-1056.9) $\mu \mathrm{g}$; mean difference (95\% CI): 249.6 (133.5 to 365.6); $p<0.001$, Figure 6a). The per-interval infused opioid dose was significantly different between groups throughout the postoperative period ( $p<0.001$, Figure $6 \mathrm{~b}$ ). It was higher in group OBIM than in group TBIM until the 6th postoperative hour and lower from the 18 th to the 48 th hours $(p \leq 0.008$, Figure $6 \mathrm{~b}$ ).

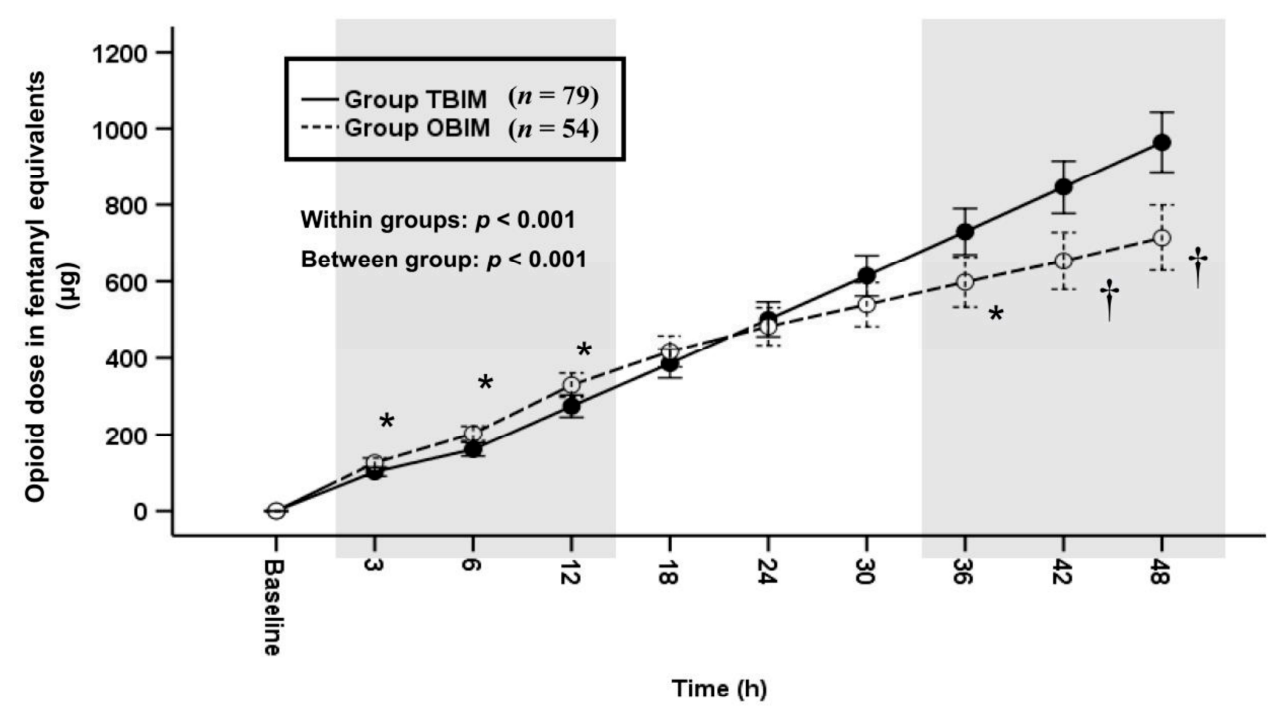

(a)

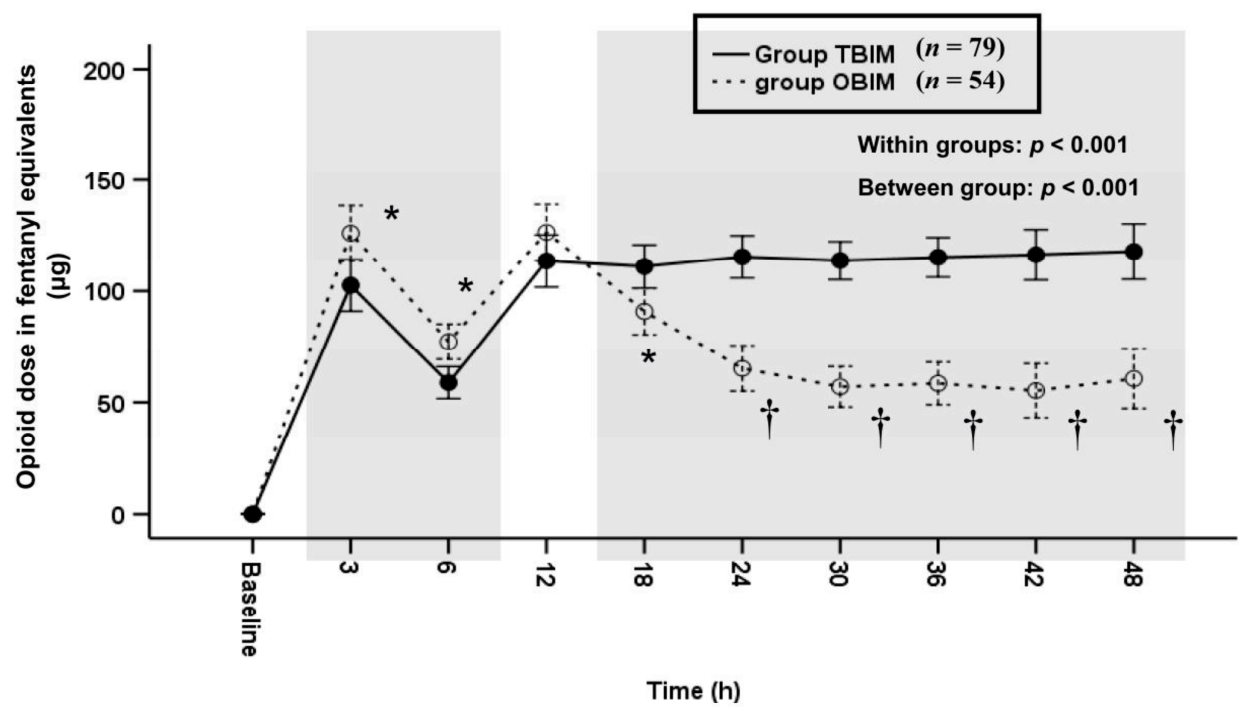

(b)

Figure 6. Time-sequential changes of cumulative (a) and per-interval (b) infused opioid dose in fentanyl equivalents. The gray boxes represent the intervals in which there were statistically significant differences between the groups. Data points and error bars represent means and 95\% confidence intervals, respectively. OBIM: optimizing background infusion mode, PCA: patient-controlled analgesia, TBIM: traditional background infusion mode. ${ }^{*}: p<0.05$ compared with group TBIM, + : $p<0.001$ compared with group TBIM. 


\subsection{Rescue Drugs and Complications}

The proportion of patients requiring rescue analgesics and antiemetics was not significantly different between the groups throughout the recovery period ( $p=0.165$ and $p=0.686$, respectively; Table 4). The specific postoperative rescue analgesics used were tramadol, diclofenac, and fentanyl, which were not significantly different between the groups throughout the recovery period ( $p \geq 0.05$, Table 5$)$. The total cumulative opioid dose after the tramadol doses were converted to opioid doses in fentanyl equivalents was not significantly different between the groups (mean: $31.5 \mu \mathrm{g}$ and $20.7 \mu \mathrm{g}$ in groups TBIM and OBIM, respectively, and mean difference (95\% CI): 10.8 ( -11.4 to 33.0$) ; p=0.339$, Table 5). The total cumulative diclofenac dose was not significantly different between the groups (mean: $1.1 \mathrm{mg}$ and $1.7 \mathrm{mg}$ in groups TBIM and OBIM, respectively, and mean difference (95\% CI): -0.5 ( -4.4 to 3.3$) ; p=0.787$, Table 5).

Table 4. Incidence of the requirement for postoperative rescue analgesics and antiemetics.

\begin{tabular}{|c|c|c|c|c|c|c|}
\hline \multirow{2}{*}{ Variables } & \multirow{2}{*}{ Groups } & \multicolumn{4}{|c|}{ Time } & \multirow{2}{*}{ Total } \\
\hline & & RR2 & $6 \mathrm{~h}$ & $24 \mathrm{~h}$ & $48 \mathrm{~h}$ & \\
\hline \multirow{3}{*}{ Analgesics } & Group TBIM $(n=79)$ & $1(1.3)$ & $14(17.7)$ & $8(10.1)$ & $6(7.6)$ & $23(29.1)$ \\
\hline & Group OBIM $(n=54)$ & $1(1.9)$ & $8(14.8)$ & $4(7.4)$ & $1(1.9)$ & $10(18.5)$ \\
\hline & $p$ value & 1.000 & 0.658 & 0.761 & 0.240 & 0.165 \\
\hline \multirow[t]{3}{*}{ Antiemetics } & Group TBIM $(n=79)$ & $0(0)$ & $0(0)$ & $3(3.8)$ & $0(0)$ & $3(3.8)$ \\
\hline & Group OBIM $(n=54)$ & $0(0)$ & $2(3.7)$ & $1(1.9)$ & $1(1.9)$ & $3(5.6)$ \\
\hline & $p$ value & - & 0.163 & 0.646 & 0.406 & 0.686 \\
\hline
\end{tabular}

Values are expressed as the number (percentage) of patients. OBIM: optimizing background infusion mode, RR2 discharge from the recovery room; TBIM: traditional background infusion mode.

Table 5. Rescue analgesics.

\begin{tabular}{|c|c|c|c|c|c|c|}
\hline \multirow{2}{*}{ Variables } & \multirow{2}{*}{\multicolumn{2}{|c|}{ Groups }} & \multicolumn{4}{|c|}{ Time } \\
\hline & & & RR2 & $6 \mathrm{~h}$ & $24 \mathrm{~h}$ & $48 \mathrm{~h}$ \\
\hline \multirow{12}{*}{ Analgesics } & & Tramadol & $1(1.3)$ & $14(17.7)$ & $7(8.9)$ & $6(7.6)$ \\
\hline & $\begin{array}{l}\text { Group IBIM } \\
(n=79)\end{array}$ & Diclofenac & $0(0)$ & $1(1.3)$ & $0(0)$ & $0(0)$ \\
\hline & & Fentanyl & $0(0)$ & $0(0)$ & $1(1.3)$ & $0(0)$ \\
\hline & & Tramadol & $1(1.9)$ & $6(11.1)$ & $8(10.1)$ & $1(1.9)$ \\
\hline & Group OBIM & Diclofenac & $0(0)$ & $1(1.9)$ & $4(7.4)$ & $0(0)$ \\
\hline & & Fentanyl & $0(0)$ & $0(0)$ & $0(0)$ & $0(0)$ \\
\hline & & $p$ value & 1.000 & 0.564 & 0.673 & 0.240 \\
\hline & & & \multicolumn{2}{|c|}{$\begin{array}{c}\text { Cumulative opioid dose } \\
\text { in fentanyl equivalents } \\
(\mu \mathrm{g})\end{array}$} & \multicolumn{2}{|c|}{$\begin{array}{c}\text { Cumulative diclofenac } \\
\text { dose (mg) }\end{array}$} \\
\hline & \multicolumn{2}{|c|}{ Group TBIM $(n=79)$} & \multicolumn{2}{|c|}{$31.5(16.3-46.7)$} & \multicolumn{2}{|c|}{$1.1(-1.1$ to 3.4$)$} \\
\hline & \multirow{2}{*}{\multicolumn{2}{|c|}{$\begin{array}{c}\text { Group OBIM }(n=54) \\
p \text { value }\end{array}$}} & \multicolumn{2}{|c|}{$20.7(5.2-36.3)$} & \multicolumn{2}{|c|}{$1.7(-1.7$ to 5.0$)$} \\
\hline & & & & & & \\
\hline & \multicolumn{2}{|c|}{ Mean difference (95\% CI) } & \multicolumn{2}{|c|}{$10.8(-11.4$ to 33.0$)$} & \multicolumn{2}{|c|}{$-0.5(-4.4$ to 3.3$)$} \\
\hline
\end{tabular}

Values are expressed as the mean (95\% confidence interval) or the number (percentage) of patients. OBIM: optimizing background infusion mode, RR2: discharge from the recovery room; TBIM: traditional background infusion mode. Intravenous tramadol (mg) was converted to intravenous fentanyl $(\mu \mathrm{g})$ according to the suggestion by the following website: https://en.wikipedia.org/wiki/equianalgesic; $1 \mathrm{mg}$ tramadol was equianalgesic to $1 \mu \mathrm{g}$ fentanyl.

\section{Discussion}

This prospective, double-blind, randomized controlled study revealed that the NRS score and bolus demand count did not differ between groups throughout the recovery period. Patients in group OBIM exhibited a higher background infusion rate before the 12th postoperative hour and a lower rate from the 12th to the 48th postoperative hours 
compared with those in group TBIM. OBIM offered adequate response to the biphasic pain pattern after laparoscopic surgery compared to TBIM. The total cumulative infused PCA volume and opioid consumption were lower in group OBIM than in group TBIM.

Many previous studies of PCA using the VFIM were conducted in patients using "computer-integrated" patient-controlled epidural analgesia (PCEA) during labor and delivery [14-17]. Their results suggested that patient satisfaction was greater in those using the computer-integrated PCEA than in those using traditional PCEA, but that the incidence of breakthrough pain and the cumulative local anesthetic consumption did not differ significantly between groups $[15,17]$. However, we are aware of only one other study in which the effect of intravenous PCA was evaluated using a similar VFIM technique to that of our study in combination with demand dosing, which was performed in patients undergoing spinal surgery [10]. In that study, the VFIM did not significantly decrease the NRS score for postoperative pain compared with the TBIM, and the NRS score decreased over time in both groups [10]. The cumulative infused PCA volume was significantly lower in the VFIM than in the TBIM group at the 24th and 48th postoperative hours. The authors assumed that this resulted from the corresponding lower bolus demand counts throughout the recovery period, with significantly lower bolus demand counts in the VFIM than in the TBIM group at the 12th and 24th postoperative hours [10]. Hence, they suggested that the VFIM could provide more efficient postoperative analgesia and reduce the cumulative infused PCA volume than the TBIM [10]. This study also demonstrated that the OBIM contributed to a reduced cumulative infused PCA volume during the first 48 postoperative hours. However, we observed no significant differences in NRS scores or bolus demand counts between the OBIM and TBIM groups. This may be explained by the relatively high proportion of patients receiving additional rescue analgesics throughout the recovery period in group TBIM, and by the relatively low pain following laparoscopic cholecystectomy compared with that following spinal surgery. If we restricted the use of additional rescue analgesics and studied patients who underwent more painful surgeries, the results may have differed.

Most patients experience a biphasic pattern of pain intensity that requires more analgesics immediately after surgery followed by less analgesics subsequently $[6,7]$. The fixed background infusion rate (TBIM) combined with bolus dosing is not enough to overcome early postoperative pain, because the lockout interval limits the rescue analgesia infusion via PCA despite frequent bolus demands $[6,7,18]$. However, the OBIM with bolus dosing has the benefit of providing more effective postoperative pain and reducing the total infused volume compared to the TBIM with bolus dosing $[10,18]$, because the OBIM increases the background infusion rate for the increased bolus demands immediately after surgery and then decreases the background infusion rate due to the absence of a bolusdosing requirement [10]. We have commonly adopted the PCA by using bolus dosing combined with the TBIM. However, the TBIM was associated with a higher incidence of insufficient postoperative analgesia and higher postoperative pain, resulting in an increase in total opioid consumption and higher postoperative bolus requirements compared to the OBIM [18]. Our study also showed that the OBIM reduced the total infused volume (total opioid consumption) even in the presence of non-significantly reduced postoperative NRS score associated with improved postoperative analgesia. So, PCA using the OBIM combined with bolus dosing can provide more sufficient postoperative analgesia and further reduce opioid consumption by decreasing the total infused volume.

Considering the biphasic postoperative-pain pattern, opioid-related adverse effects are a major concern in patients using PCA. The OBIM PCA may result in adverse effects because of an increased background infusion rate and an increased bolus demand due to high levels of pain experienced immediately after surgery. On the other hand, the TBIM PCA may result in an unnecessary infusion of opioids in patients that do not require active pain control beyond the acute period of postoperative pain [10]. This study revealed that postoperative nausea requiring antiemetics mainly occurred before the sixth postoperative 
hour in the OBIM group (3.7\%), and after the sixth postoperative hour in the TBIM group (3.8\%; Table 4). No other adverse effects were observed.

Lee at al. [10] documented that the overall incidence of PONV requiring antiemetics was lower in the OBIM group (18\%) compared to the TBIM group (33\%); in contrast, in this study it was higher in the OBIM group (5.6\%) than the TBIM group (3.8\%; Table 4). This discrepancy has several possible explanations. First, Lee at al. [10] used PCA devices with opioids alone, while we combined opioids and antiemetics. Our use of premixed antiemetics probably contributed to reducing the overall incidence of PONV in both groups compared to the study by Lee et al. [10]. Second, we did not confirm whether the PONV was directly related to the administered opioid dose as we did not record the incidence of PONV at each time interval. In this study, we enrolled patients who underwent laparoscopic surgery, an important risk factor for PONV. Even though the premixed antiemetics reduced the overall incidence of PONV, the risk of PONV was probably increased by the increased background infusion rate of opioids during the acute period in the OBIM group, resulting in a higher incidence of PONV in this group than in the TBIM group.

The major limitation of this study was the drop-out rate (34.8\%), which was much higher than expected $(15 \%)$. The causes included data loss when downloading from the PCA device (18.2\%), early termination of the PCA (9.8\%), and device-setting errors (6.4\%). First, even though we allowed ample time for the RP to be trained in the setup of the PCA, we had to exclude $2.9 \%$ of patients in group TBIM and $9.8 \%$ in group OBIM due to setting errors. OBIM requires, in addition to the setup of the TBIM, the setup of conditions and sizes for increases and decreases in the background infusion rate as well as the maximum and minimum allowable background infusion rates. This complex setup, combined with unfamiliarity with the OBIM of the PCA device, requires ample training time to prepare and operate the device in order to reduce setup and operation errors [10]. Second, some patients who underwent laparoscopic surgeries were discharged early due to low levels of postoperative pain and a quick recovery. Third, part of the PCA data were lost as we overlooked the fact that the data is erased when the device is powered down. Therefore, a more secure system should be implemented for downloading data from the device in future studies.

Another limitation of this study is that we did not adopt multimodal pain management protocols including preemptive analgesia, non-steroidal anti-inflammatory drugs, gabapentinoids, acetaminophen, muscle relaxants, ketamine, neuro-axial blockade, and local infiltrative anesthetic. [19]. Therefore, we cannot conclude that OBIM will be able to provide more effective postoperative analgesia in the context of applying a multimodal pain management protocol with PCA.

\section{Conclusions}

The OBIM reduces cumulative PCA volume and opioid consumption by responding more effectively to postoperative pain compared to the TBIM, while yielding comparable postoperative analgesia and bolus demand in patients undergoing laparoscopic cholecystectomy. Further studies are required to determine the efficacy of the OBIM in different types of surgery and degrees of postoperative pain.

Author Contributions: Conceptualization, K.T.J., K.Y.S. and S.H.K.; methodology, K.T.J., S.U.K. and S.H.K.; software, S.H.K.; validation, S.H.K.; formal analysis, S.H.K.; investigation, K.T.J., K.Y.S. and S.U.K.; resources, S.H.K.; data curation, S.H.K.; writing—original draft preparation, K.T.J.; writingreview and editing, S.H.K.; visualization, K.T.J., S.U.K. and S.H.K.; supervision, S.H.K.; project administration, S.U.K. and S.H.K.; funding acquisition, S.H.K. All authors have read and agreed to the published version of the manuscript.

Funding: The research and the APC were supported by research funds from Chosun University Hospital (Donggu, Gwangju, Korea), 2018. 
Institutional Review Board Statement: This prospective, randomized, controlled, double-blind study was approved by the Institutional Review Board of Chosun University Hospital (Chosun 201802-011) on 6 March 2018, and was prospectively registered with the Clinical Research Information Service (CRIS: 75 https:/ / cris.nih.go.kr/, ref: KCT0002777) on 5 April 2018. It was conducted according to the Declaration of Helsinki of 1964 and all of its subsequent revisions.

Informed Consent Statement: Written informed consent was obtained from all participants after a thorough explanation of the purpose of the study.

Data Availability Statement: The data presented in this study are available on request from the corresponding author, through institutional review board, and reviewers. The data are not publicly available due to restrictions of obtaining approval from the IRB for the disclosure of data. If anyone requires our data of this study, please do not hesitate to contact the corresponding author.

Conflicts of Interest: The authors declare no conflict of interest that may have influenced either the conduct or the presentation of the research. Chosun University Hospital, as the funder, had no role in the design of the study; in the collection, analyses, or interpretation of data; in the writing of the manuscript, or in the decision to publish the results.

\section{References}

1. Grass, J.A. Patient-controlled analgesia. Anesth. Analg. 2005, 101, S44-S61. [CrossRef]

2. Gepstein, R.; Arinzon, Z.; Folman, Y.; Shuval, I.; Shabat, S. Efficacy and complications of patient-controlled analgesia treatment after spinal surgery. Surg. Neurol. 2007, 67, 360-366. [CrossRef] [PubMed]

3. McNicol, E.D.; Ferguson, M.C.; Hudcova, J. Patient controlled opioid analgesia versus non-patient controlled opioid analgesia for postoperative pain. Cochrane Database Syst. Rev. 2015. [CrossRef] [PubMed]

4. Kye-Min, K. Analysis of the current state of postoperative patient-controlled analgesia in Korea. Anesthesiol. Pain Med. 2016, 11, 28-35.

5. Lehmann, K.A. Recent developments in patient-controlled analgesia. J. Pain Symptom Manag. 2005, 29, S72-S89. [CrossRef] [PubMed]

6. Nielsen, R.V.; Fomsgaard, J.S.; Dahl, J.B.; Mathiesen, O. Insufficient pain management after spine surgery. Dan. Med. J. 2014, 61, A4835. [PubMed]

7. Parker, R.K.; Holtmann, B.; White, P.F. Effects of a nighttime opioid infusion with PCA therapy on patient comfort and analgesic requirements after abdominal hysterectomy. Anesthesiology 1992, 76, 362-367. [CrossRef] [PubMed]

8. Smythe, M.A.; Zak, M.B.; O’Donnell, M.P.; Schad, R.F.; Dmuchowski, C.F. Patient-controlled analgesia versus patient-controlled analgesia plus continuous infusion after hip replacement surgery. Ann. Pharmacother. 1996, 30, 224-227. [CrossRef] [PubMed]

9. Chen, W.H.; Liu, K.; Tan, P.H.; Chia, Y.Y. Effects of postoperative background PCA morphine infusion on pain management and related side effects in patients undergoing abdominal hysterectomy. J. Clin. Anesth. 2011, 23, 124-129. [CrossRef] [PubMed]

10. Lee, S.H.; Baek, C.W.; Kang, H.; Park, Y.H.; Choi, G.J.; Jung, Y.H.; Woo, Y.C. A comparison of 2 intravenous patient-controlled analgesia modes after spinal fusion surgery: Constant-rate background infusion versus variable-rate feedback infusion, a randomized controlled trial. Medicine 2019, 98, e14753. [CrossRef] [PubMed]

11. Sng, B.L.; Zhang, Q.; Leong, W.L.; Ocampo, C.; Assam, P.N.; Sia, A.T. Incidence and characteristics of breakthrough pain in parturients using computer-integrated patient-controlled epidural analgesia. J. Clin. Anesth. 2015, 27, 277-284. [CrossRef] [PubMed]

12. Kim, D.K.; Yoon, S.H.; Kim, J.Y.; Oh, C.H.; Jung, J.K.; Kim, J. Comparison of the Effects of Sufentanil and Fentanyl Intravenous Patient Controlled Analgesia after Lumbar Fusion. J. Korean Neurosurg. Soc. 2017, 60, 54-59. [CrossRef] [PubMed]

13. Faul, F.; Erdfelder, E.; Lang, A.G.; Buchner, A. G*Power 3: A flexible statistical power analysis program for the social, behavioral, and biomedical sciences. Behav. Res. Methods 2007, 39, 175-191. [CrossRef] [PubMed]

14. Sng, B.L.; Woo, D.; Leong, W.L.; Wang, H.; Assam, P.N.; Sia, A.T. Comparison of computer-integrated patient-controlled epidural analgesia with no initial basal infusion versus moderate basal infusion for labor and delivery: A randomized controlled trial. J. Anaesthesiol. Clin. Pharmacol. 2014, 30, 496-501. [CrossRef] [PubMed]

15. Sng, B.L.; Sia, A.T.; Lim, Y.; Woo, D.; Ocampo, C. Comparison of computer-integrated patient-controlled epidural analgesia and patient-controlled epidural analgesia with a basal infusion for labour and delivery. Anaesth. Intensive Care 2009, $37,46-53$. [CrossRef] [PubMed]

16. Sia, A.T.; Lim, Y.; Ocampo, C.E. Computer-integrated patient-controlled epidural analgesia: A preliminary study on a novel approach of providing pain relief in labour. Singapore Med. J. 2006, 47, 951-956. [PubMed]

17. Lim, Y.; Sia, A.T.; Ocampo, C.E. Comparison of computer integrated patient controlled epidural analgesia vs. conventional patient controlled epidural analgesia for pain relief in labour. Anaesthesia 2006, 61, 339-344. [CrossRef] [PubMed]

18. Hwang, J.; Min, S.K.; Chae, Y.J.; Lim, G.M.; Joe, H.B. Continuous Fentanyl Background Infusion Regimen Optimised by PatientControlled Analgesia for Acute Postoperative Pain Management: A Randomised Controlled Trial. J. Clin. Med. 2020, 9, 211. [CrossRef]

19. Devin, C.J.; McGirt, M.J. Best evidence in multimodal pain management in spine surgery and means of assessing postoperative pain and functional outcomes. J. Clin. Neurosci. 2015, 22, 930-938. [CrossRef] [PubMed] 